\title{
Microstructure and mechanical properties of a heat resistant nickel base superalloy heavily alloyed with substitution elements
}

\author{
V. M. Imayev ${ }^{1}$, Sh. Kh. Mukhtarov ${ }^{\dagger, 1}$, A. V. Logunov², A. A. Ganeev ${ }^{1}$, R. V. Shakhov ${ }^{1}$, \\ R. I. Zainullin ${ }^{1}$, N. Yu. Parkhimovich ${ }^{1}$, R. M. Imayev ${ }^{1}$ \\ ${ }^{\dagger}$ shamilm@imsp.ru
}

${ }^{1}$ Institute for Metals Superplasticity Problems of RAS, Ufa, 450001, Russia ${ }^{2}$ PJSC “UEC-Saturn”, Rybinsk, 152903, Russia

The present work is devoted to a study of the microstructure and mechanical properties of a new nickel base superalloy Ni-14(Al, Ta)-30.7(Co, Cr, W, Hf) (wt.\%) intended for use as a die material and probably a structural material for gas turbine engines (GTEs). The superalloy contains about $70 \mathrm{vol} . \%$ of the $\gamma^{\prime}\left(\mathrm{Ni}_{3} \mathrm{Al}\right)$ phase and about 3 vol.\% of carbides and topologically close-packed (TCP) phases. Before hot forging, the superalloy workpiece was subjected to homogenization annealing followed by slow furnace cooling that led to coarsening of the $\gamma^{\prime}$ phase. The obtained microstructural condition was used to evaluate the partitioning coefficients $k_{\gamma / \gamma^{\prime}}$ for alloying elements by energy dispersive X-Ray (EDS) analysis. EDS analysis was also performed for TCP phases. It was revealed that the TCP phases contained a higher amount of Ta, W, and Hf. Compression tests carried out for the cast and heat treated condition showed that the new superalloy had the yield strength values at $1100-1200^{\circ} \mathrm{C}$ comparable with those obtained for the VKNA superalloys based on the $\gamma^{\prime}$ phase; at $1000^{\circ} \mathrm{C}$ the yield strength was found to be much higher than that of the VKNA superalloys. In the wrought conditions, the ductility and strength properties at $20-750^{\circ} \mathrm{C}$ were appreciably higher than those in the cast and heat treated condition. At the same time, the studied microstructural conditions of the superalloy showed a similar creep rupture life at $650-850^{\circ} \mathrm{C}$. The obtained mechanical properties show that the new superalloy can be considered as an inexpensive structural material for GTEs.

Keywords: nickel base superalloy, heat treatment, hot forging, microstructure, mechanical properties.

УДК: 669.245

\section{Микроструктура и механические свойства жаропрочного никелевого сплава, высоколегированного элементами замещения}

\author{
Имаев В. М. ${ }^{1}$, Мухтаров Ш. Х. ${ }^{\dagger, 1}$, Логунов А. В. ${ }^{2}$, Ганеев А. А. ${ }^{1}$, Шахов Р. В. ${ }^{1}$, \\ Зайнуллин Р. И. ${ }^{1}$, Пархимович Н. Ю. ${ }^{1}$, Имаев Р. М. ${ }^{1}$ \\ ${ }^{1}$ Институт проблем сверхпластичности металлов РАН, Уфа, 450001, Россия \\ ${ }^{2}$ ПАО «ОДК-Сатурн», Рыбинск, 152903, Россия
}

Работа посвящена исследованию микроструктуры и механических свойств экспериментального жаропрочного никелевого сплава $\mathrm{Ni}-14(\mathrm{Al}, \mathrm{Ta})-30.7(\mathrm{Co}, \mathrm{Cr}, \mathrm{W}, \mathrm{Hf})$ (вес.\%), предполагаемого для использования в качестве материала штампового инструмента и, возможно, конструкционного материала для газотурбинного двигателя (ГТД). Сплав содержал около 70 об.\% $\gamma^{\prime}\left(\mathrm{Ni}_{3} \mathrm{Al}\right)$-фазы и около 3 об.\% карбидов и топологически плотноупакованных (ТПУ) фаз. Перед деформационной обработкой заготовку сплава подвергли гомогенизационному отжигу с последующим медленным охлаждением в печи, что привело к формированию крупной $\gamma^{\prime}$-фазы. Полученное состояние было использовано для оценки коэффициентов распределения легирующих элементов $k_{\gamma / \gamma^{\prime}}$ с помощью энергодисперсионного микрорентгеноспектрального (EDS) анализа. EDS анализ был также проведен для ТПУ фаз. Обнаружено, что ТПУ фазы содержали повышенное количество Та, W и Hf. Испытания образцов сплава на сжатие в литом состоянии 
после термической обработки показали, что новый сплав при температурах $1100-1200^{\circ} \mathrm{C}$ имеет предел текучести сравнимый с пределом текучести интерметаллидных сплавов типа ВКНА на основе $\gamma^{\prime}$-фазы, а при $1000^{\circ} \mathrm{C}$ значительно превышающий. Деформационная обработка обеспечила значительное повышение пластических и прочностных свойств сплава при температурах $20-750^{\circ} \mathrm{C}$. В то же время, длительная прочность при $650-850^{\circ} \mathrm{C}$ в исследуемых состояниях сплава оказалась близкой. Полученные механические свойства показывают, что новый сплав может рассматриваться, как недорогой конструкционный материал для ГТД.

Ключевые слова: жаропрочный никелевый сплав, термическая обработка, деформационная обработка, микроструктура, механические свойства.

\section{1. Введение}

Детали ответственного назначения, выполненные из жаропрочных никелевых сплавов, изготовленных литьем или с помощью порошковой металлургии, обычно подвергаются термической и деформационной обработке с использованием горячей экструзии, ковки или раскатки [1-4]. Горячая деформация нацелена на устранение дендритной ликвации (в случае литья), измельчение структуры за счет развития рекристаллизационных процессов и получение формы заготовки, приближенной к форме конечной детали. Деформационная обработка особенно актуальна при изготовлении сложнопрофильных деталей, таких как диски для газотурбинного двигателя (ГТД), поскольку требования по эксплуатационным свойствам, предъявляемые к деталям ГТД, постоянно растут в сторону повышенных температур и нагрузок. Между тем разработка экономически целесообразной технологии деформационной обработки сложнопрофильных заготовок из таких жаропрочных сплавов как ВЖ175, ВВ751П, СДЖС-15 является сложной научно-технической проблемой, поскольку ковка или раскатка таких жаропрочных сплавов должна осуществляться при температурах $1100-1200^{\circ} \mathrm{C}$, что требует соответствующего штампового материала. В качестве штампового материала применяют интерметаллидные сплавы типа ВКНА на основе фазы $\gamma^{\prime}\left(\mathrm{Ni}_{3} \mathrm{Al}\right)$, имеющие стабильную структуру и не требующие защитной атмосферы в ходе горячей штамповки при указанных температурах. Однако сплавы типа ВКНА имеют довольно низкие значения предела текучести при температурах $1000^{\circ} \mathrm{C}$ и ниже $[5,6]$. Представляет интерес разработка недорогого штампового материала альтернативного ВКНА-сплавам и имеющего хорошие литейные свойства, что позволит с помощью литья и последующей механической обработки изготавливать массивные штампы, раскатные ролики и др.

Настоящая работа является продолжением ранее выполненной работы [7]. Кроме испытаний на сжатие в литом состоянии для нового сплава были проведены испытания на растяжение и высокотемпературную длительную прочность после горячей штамповки и термической обработки.

\section{2. Материал и методики}

Слитки размером $\varnothing 45 \times 270$ мм сплава номинального состава $\mathrm{Ni}-14(\mathrm{Al}, \mathrm{Ta})-30.7(\mathrm{Co}, \mathrm{Cr}, \mathrm{W}, \mathrm{Hf})$ (вес.\%) были изготовлены с помощью вакуумно-индукционной плавки. Реальный состав сплава, измеренный с помощью энергодисперсионного микрорентгеноспектрального (EDS) анализа, незначительно отличался от номинального состава. Сплав содержал повышенное количество $\gamma^{\prime}$-образующих $\mathrm{Al}$ и Та и элементов замещения в $\gamma$-фазе (Co, Cr, W, Hf). Кроме указанных элементов, в сплаве содержался углерод в количестве около 0.15 вес.\%, а также Ce, Zr и В в количестве 0.06 вес.\%. Температура полного растворения $\gamma^{\prime}$-фазы, определенная с помощью закалочных экспериментов, составила $T_{\mathrm{s}} \approx 1260^{\circ} \mathrm{C}$.

Перед испытаниями на сжатие и растяжение литые заготовки сплава подвергли гомогенизационному отжигу, обработке на твердый раствор при $1250^{\circ} \mathrm{C}$ с последующим охлаждением на воздухе со скоростью около $10^{\circ} \mathrm{C} /$ сек и двухстадийному старению при 1000 и $880^{\circ} \mathrm{C}$ (Состояние 1). Состояния 2 и 3 были получены с помощью деформационной обработки. Перед деформационной обработкой заготовки сплава подвергли гомогенизационному отжигу и медленному охлаждению в печи со скоростью $25^{\circ} \mathrm{C} /$ ч ч что привело к укрупнению $\gamma^{\prime}$-фазы и уменьшению объемной доли дисперсной $\gamma^{\prime}$-фазы, что, в свою очередь, способствовало повышению деформационной способности сплава. После преддеформационной термической обработки заготовки сплава поместили в толстостенный контейнер и подвергли дробной деформации сжатием с промежуточными отжигами на общую степень деформации $e \approx 1.4$. Температура нагрева заготовки и промежуточных отжигов составляла $1210^{\circ} \mathrm{C}$, температура штампового инструмента $-930^{\circ} \mathrm{C}$. Штампованные заготовки сплава извлекали из штампового блока в течение не более 10 сек и подвергали принудительному охлаждению на воздухе со скоростью около $10^{\circ} \mathrm{C} /$ сек. После этого штамповки старили при 1000 и $880^{\circ} \mathrm{C}$ (состояние 2) или подвергали обработке на твердый раствор при температуре $1210^{\circ} \mathrm{C}$ с охлаждением на воздухе со скоростью около $10^{\circ} \mathrm{C} /$ сек с последующим старением при 860 и $750^{\circ} \mathrm{C}$ (состояние 3 ). Температура старения в состоянии 3 была снижена, чтобы уменьшить размер вторичной $\gamma^{\prime}$-фазы. Горячую штамповку осуществляли на гидравлическом прессе с максимальным усилием 6300 кН, оснащенном нагревательным штамповым блоком.

Микроструктурные исследования были выполнены на сканирующем электронном микроскопе Mira-3 Tescan в режиме вторичных (SE) или обратно-рассеянных (BSE) электронов, оборудованном приставкой для микрорентгеноспектрального энергодисперсионного (EDS) анализа. EDS-анализ был проведен для литого состояния после гомогенизационного отжига и последующего медленного охлаждения со скоростью $25^{\circ} \mathrm{C} /$. Медленное охлаждение способствовало укрупнению $\gamma^{\prime}$-фазы и уменьшению 
объемной доли дисперсной $\gamma^{\prime}$-фазы, что позволило оценить количественно распределение легирующих элементов между $\gamma$ - и $\gamma^{\prime}$-фазой. В общей сложности было выполнено не менее 50 измерений для $\gamma$ зерен и частиц $\gamma^{\prime}$-фазы, на основе чего были рассчитаны средние коэффициенты распределения $\left(k_{\gamma / \gamma^{\prime}}\right)$ легирующих элементов.

Испытания на сжатие проводили для состояния 1 сплава. Для этого были вырезаны образцы $\varnothing 8$ мм $\times 12$ мм. Испытания проводили при $T=1000-1200^{\circ} \mathrm{C}$ с начальной скоростью деформации $\dot{\varepsilon} \approx 10^{-3} \mathrm{c}^{-1}$ на степень деформации $\varepsilon=10 \%$. Кривые истинное напряжение - истинная деформация были построены сучетом равномерного увеличения поперечного сечения образцов в процессе сжатия. По кривым определяли предел текучести при сжатии $\left(\sigma_{0.2}\right)$.

Испытания на растяжение были выполнены для состояний сплава 1-3. Для этого использовали плоские образцы с размерами рабочей части $10 \times 3 \times 2 \mathrm{mм}^{3}$. Испытания проводили при 20 и $650-850^{\circ} \mathrm{C}$ со скоростью деформирования $0.5 \mathrm{mм} /$ мин, что соответствовало начальной скорости деформации $\dot{\varepsilon} \approx 8.3 \times 10^{-4} \mathrm{c}^{-1}$.

Испытания на длительную прочность были выполнены при температурах $650-850^{\circ} \mathrm{C}$ и нагрузках в диапазоне 300-1000 МПа. Использовали испытательную машину ATS2330. Зависимости Ларсона-Миллера (LMP) для прогнозирования продолжительности испытания образцов сплава до разрушения рассчитывали по формуле [8-10]: LMP $=T \times\left[\log t_{\text {rup }}+C_{\mathrm{LM}}\right]$, где $T-$ абсолютная температура испытания на длительную прочность, $t_{\text {rup }}$ - время до разрушения образца и $C_{\mathrm{LM}}-$ постоянная Ларсона-Миллера, которая была принята как $20[9,10]$. Bce механические испытания проводили на воздухе. Образцы перед испытаниями механически полировали.

\section{3. Результаты и обсуждение}

\section{1. Влияние обработки на микроструктуру сплава}

На Рис. 1 представлена микроструктура сплава в состояниях 1-3. В состоянии 1 размер $\gamma$ зерен составил $d_{\gamma}=100-500$ мкм, размер первичной $\gamma^{\prime}$-фазы $-d_{\gamma^{\prime}}=1-5$ мкм, средний размер вторичной $\gamma^{\prime}$-фазы, выделившейся при охлаждении на воздухе и старении $-d_{\gamma^{\prime}} \approx 0.2$ мкм (Рис. $\left.1 \mathrm{a}, \mathrm{d}\right)$. Объемная доля первичной $\gamma^{\prime}$-фазы была не более $5 \%$. Общая объемная доля $\gamma^{\prime}$-фазы была определена в состоянии после гомогенизационного отжига и медленного охлаждения; она составила около 70\%. В микроструктуре присутствовали также карбиды и топологически плотноупакованные (ТПУ) фазы, их объемная доля не превышала $3 \%$.

Горячая штамповка с промежуточными отжигами привела к развитию рекристаллизационных процессов и измельчению исходной крупнозернистой литой структуры. В состоянии 2 размер рекристаллизованных $\gamma$ зерен составил $d_{\gamma}=2-50$ мкм (Рис. 1b). В микроструктуре отмечались также отдельные нерекристаллизованные $\gamma$ зерна с размером до 100 мкм. Первичная $\gamma^{\prime}$-фаза, не растворившаяся в процессе штамповки, занимала около 30 об.\%, её размер составил $d_{\gamma^{\prime}}=1-10$ мкм (Рис. 1е). Дисперсная вторичная $\gamma^{\prime}$-фаза выделилась при охлаждении штамповки и старении. Средний размер вторичной $\gamma^{\prime}$-фазы составил $d_{\gamma^{\prime}} \approx 0.2$ мкм. Объемная доля карбидов и ТПУ фаз после горячей штамповки и старения немного выросла и составила $3.4 \%$.

Обработка на твердый раствор (состояние 3) привела к увеличению размера мелких рекристаллизованных

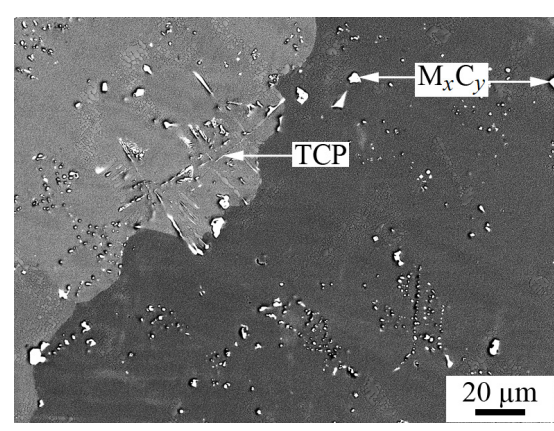

a

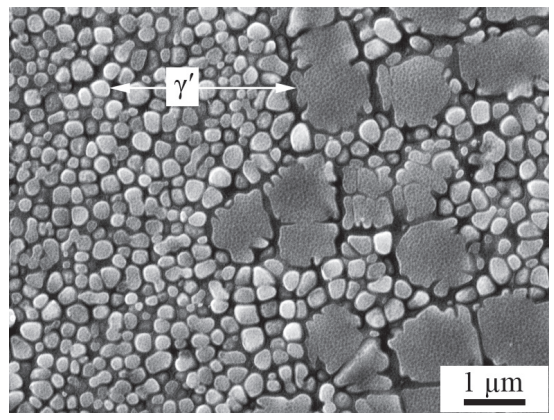

d

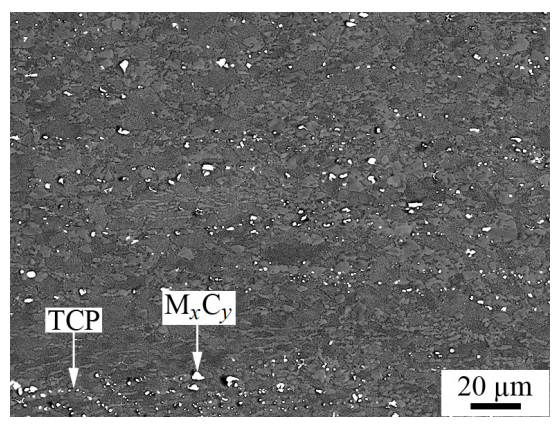

b

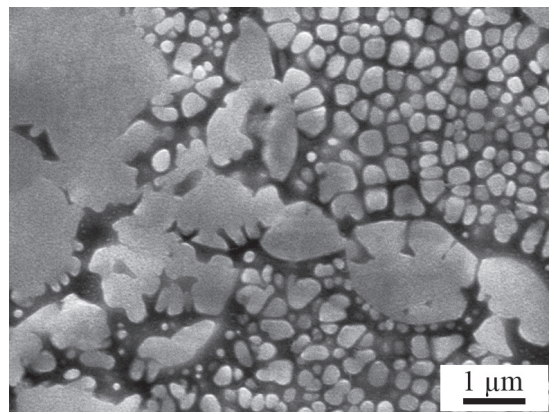

e

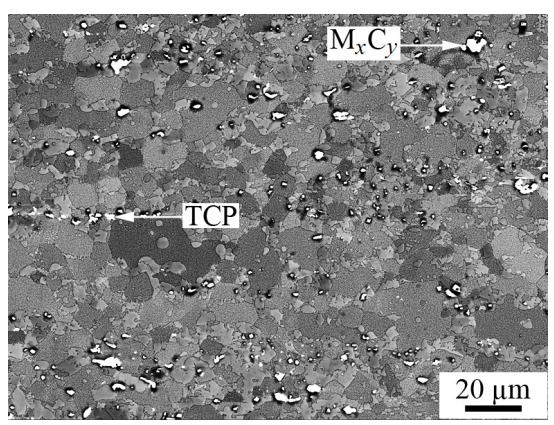

c

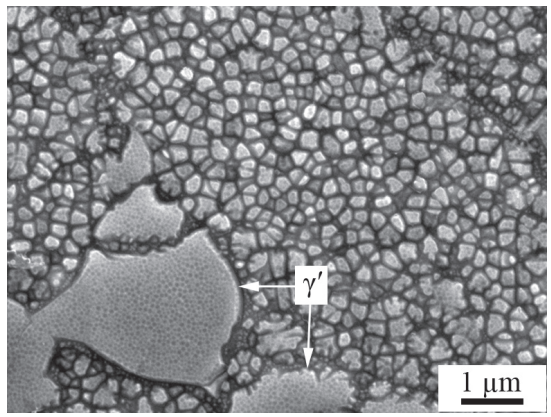

f

Рис. 1. Микроструктура сплава $\mathrm{Ni}-14(\mathrm{Al}, \mathrm{Ta})-30.7(\mathrm{Co}, \mathrm{Cr}, \mathrm{W}, \mathrm{Hf})$ : состояние 1 (a, d), состояние 2 (b, e), состояние 3 (с, f). Стрелки указывают на первичную, вторичную $\gamma^{\prime}$ фазу, карбиды $\left(\mathrm{M}_{x} \mathrm{C}_{y}\right)$ и ТПУ фазы (BSE - a, b, c; SE - d, c, f).

Fig. 1. Microstructural images of the Ni-14(Al, Ta)-30.7(Co, Cr, W, Hf) superalloy: condition 1 (a, d), condition 2 (b, e), condition 3 (c, f). Arrows indicate the primary and secondary $\gamma^{\prime}$ phase, the carbides $\left(\mathrm{M}_{x} \mathrm{C}_{y}\right)$ and the TCP phases (BSE - a, b, c; SE $\left.-\mathrm{d}, \mathrm{c}, \mathrm{f}\right)$. 
$\gamma$ зерен, а также к уменьшению объемной доли первичной $\gamma^{\prime}$-фазы до $25 \%$ и её размера до $d_{\gamma^{\prime}}=3-5$ мкм (Рис. $1 \mathrm{c}, \mathrm{f}$ ). Уменьшение объемной доли и размера первичной $\gamma^{\prime}$-фазы по сравнению с состоянием 2 указывает на то, что обработка на твердый раствор привела к частичному растворению первичной $\gamma^{\prime}$-фазы. Средний размер вторичной $\gamma^{\prime}$-фазы в состоянии 3 составил $d_{\gamma^{\prime}} \approx 0.15$ мкм, что меньше, чем в состояниях 1 и 2 (Рис. $1 \mathrm{~d}, \mathrm{e}, \mathrm{f}$ ). По всей видимости, меньший размер вторичной $\gamma^{\prime}$-фазы в состоянии 3 указывает на то, что старение при более низкой температуре $\left(860\right.$ и $\left.750^{\circ} \mathrm{C}\right)$ способствовало формированию более дисперсной вторичной $\gamma^{\prime}$-фазы.

\section{2. Распределение легируюших элементов в сплаве, измеренное с помощью EDS-анализа}

Литое состояние сплава, подвергнутое гомогенизационному отжигу и последующему медленному охлаждению в печи, было использовано для оценки коэффициента распределения легирующих элементов. Как отмечалось, медленное охлаждение привело к укрупнению $\gamma^{\prime}$-фазы и значительному уменьшению объемной доли дисперсной $\gamma^{\prime}$-фазы. EDS-анализ был выполнен для участков $\gamma$ зерен, свободных от дисперсных выделений $\gamma^{\prime}$-фазы, а также для крупных частиц $\gamma^{\prime}$-фазы.

В Табл. 1 представлены среднее содержание легирующих элементов в $\gamma$ - и $\gamma^{\prime}$-фазе и средние рассчитанные коэффициенты распределения $k_{\gamma / \gamma^{\prime}}$ легирующих элементов. Следует ожидать, что наибольшему твердорастворному упрочнению (на единицу концентрации легирующего элемента) в $\gamma$ - и $\gamma^{\prime}$-фазе будет отвечать, соответственно, наибольший и наименьший коэффициент $k_{\gamma / \gamma}$. Исходя из этого, наибольшее твердорастворное упрочнение (на единицу концентрации) в $\gamma$ - и $\gamma^{\prime}$-фазе обеспечивают, соответственно, Cr, W, Со и Та. Распределение Hf не анализировали из-за его низкого содержания в сплаве. Кроме того, EDS-анализ обнаружил, что ТПУ фазы, помимо Та и W были обогащены Hf, что объясняет близкое к нулю содержание Нf в $\gamma$ - и $\gamma^{\prime}$-фазе.

\section{3. Механические свойства сплава при сжатии}

На Рис. 2 представлены кривые истинное напряжение истинная деформация, полученные в результате испытаний образцов сплава на сжатие при $T=1000-1200^{\circ} \mathrm{C}$. Напряжение течения уменьшалось с увеличением температуры испытания. Важно отметить, что напряжение течения почти не менялось в зависимости от величины деформации (после выхода на установившееся течение) при всех температурах испытания. Это указывает на высокую стабильность микроструктуры сплава. Предел текучести сплава при сжатии при температуре $1000^{\circ} \mathrm{C}$ оказался значительно выше в сравнении с ВКНА-сплавами и традиционным штамповым никелевым сплавом ЖС6У (Табл. 2). При температурах $1100-1200^{\circ} \mathrm{C}$ предел текучести при сжатии исследуемого сплава оказался близок к пределу текучести ВКНАсплавов $[6,11]$.

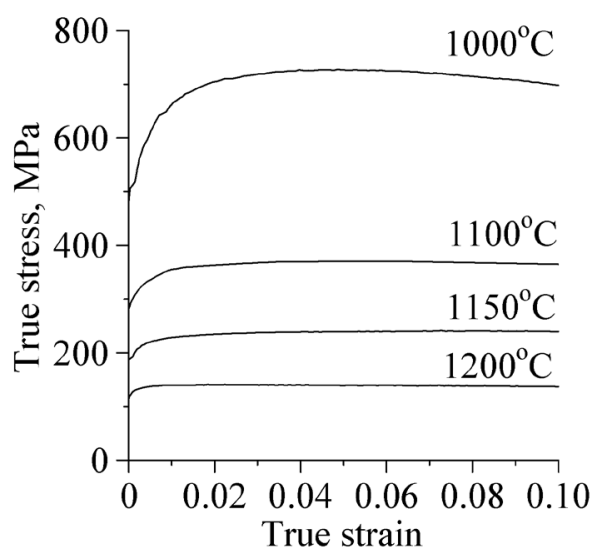

Рис. 2. Кривые истинное напряжение - истинная деформация, полученные в результате испытаний на сжатие образцов сплава в состоянии $1\left(\dot{\varepsilon} \approx 10^{-3} \mathrm{c}^{-1}\right)$.

Fig. 2. True stress - true strain curves obtained as a result of compression tests of the superalloy specimens in condition 1 $\left(\dot{\varepsilon} \approx 10^{-3} \mathrm{~s}^{-1}\right)$.

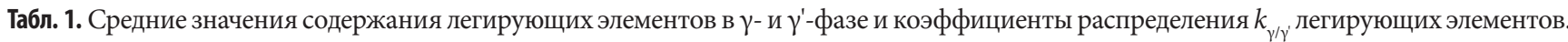
Table 1. The average values of alloying elements in the $\gamma$ - and $\gamma^{\prime}$-phase and the partition coefficients $k_{\gamma / \gamma^{\prime}}$ obtained for the alloying elements.

\begin{tabular}{|c|c|c|c|c|c|c|c|}
\hline Analyzed area / partition coefficient & $\mathrm{Al}$ & $\mathrm{Ta}$ & $\mathrm{Ni}$ & $\mathrm{Cr}$ & $\mathrm{Co}$ & $\mathrm{W}$ & $\mathrm{Hf}$ \\
\hline$\gamma$-матрица / $\gamma$-matrix & 3.66 & 3.71 & 50.74 & 10.97 & 18.6 & 12.32 & - \\
\hline$\gamma^{\prime}$-фаза / $\gamma^{\prime}$-phase & 7.20 & 12.48 & 60.14 & 2.66 & 10.7 & 6.82 & - \\
\hline$k_{\gamma / \gamma^{\prime}}$ & 0.508 & 0.297 & 0.844 & 4.124 & 1.738 & 1.806 & - \\
\hline
\end{tabular}

Табл. 2. Температурная зависимость предела текучести (в МПа) при сжатии сплава Ni-14(Al, Ta)-30.7(Co, Cr, W, Hf) в состоянии 1 в сравнении с другими жаропрочными никелевыми сплавами, используемыми в качестве штамповых материалов.

Table 2. Temperature dependence of the compression yield strength (in $\mathrm{MPa}$ ) of the Ni-14(Al, Ta)-30.7(Co, Cr, W, Hf) superalloy in condition 1 in comparison with those of other nickel-based superalloys used as die materials.

\begin{tabular}{|c|c|c|c|c|}
\hline \multirow{2}{*}{ Сплав / Superalloy } & \multicolumn{3}{|c|}{ Температура испытания, ${ }^{\circ} \mathrm{C} /$ Test temperature, ${ }^{\circ} \mathrm{C}$} \\
\cline { 2 - 5 } & 1000 & 1100 & 1150 & 1200 \\
\hline Ni-14(Al, Ta)-30.7(Co, Cr, W, Hf) & $705 \pm 21$ & $360 \pm 11$ & $234 \pm 7$ & $140 \pm 5$ \\
\hline BКHA-4 / VKNA-4 [6] & 290 & 215 & - & 115 \\
\hline BKHA-1B / VKNA-1V [6] & 430 & 350 & - & 185 \\
\hline BKHA-1BУ / VKNA-1VU [6] & 575 & 430 & - & 145 \\
\hline BКHA-4Y / VKNA-4U [6] & 430 & 370 & - & 150 \\
\hline BКHA-25 / VKNA-25 [6] & 510 & 370 & - & - \\
\hline ЖС6У / ZhS6U [11] & 510 & 298 & - & 170 \\
\hline
\end{tabular}




\section{4. Механические свойства сплава при растяжении}

На Рис. 3 представлены результаты испытаний сплава на растяжение в состояниях 1-3. Измельчение структуры в результате горячей штамповки обеспечило значительное повышение пластичности и прочности сплава при температурах $20-750^{\circ} \mathrm{C}$. При температуре $850^{\circ} \mathrm{C}$ прочностные свойства оказались немного выше в состоянии с крупнозернистой структурой (состоянии 1). Это объясняется, по-видимому, развитием релаксационных процессов, в частности, проскальзывания по межзеренным/межфазным границам при этой температуре в мелкозернистых состояниях 2 и 3. Интенсивнее указанные процессы при $850^{\circ} \mathrm{C}$ развивались в наиболее мелкозернистом состоянии, что объясняет пониженные прочностные свойства при этой температуре в состоянии 2 по сравнению с состоянием 3. Причины повышенной пластичности сплава в состоянии 3 в сравнении с состоянием 2 при 650 и $750^{\circ} \mathrm{C}$, как и причины значительного снижения пластичности сплава с повышением температуры до конца не ясны. Следует отметить, что снижение пластичности с повышением температуры испытания наблюдали и в др. никелевых сплавах [12-14].

\section{5. Высокотемпературная длительная прочность}

На Рис. 4 представлены зависимости напряжения от параметра Ларсона-Миллера, для состояний 1-3 сплава, которые были построены по результатам испытаний образцов на длительную прочность в диапазоне $650-850^{\circ} \mathrm{C} / 300-1000$ МПа. В целом, результаты испытаний показали, что сплав в состояниях 1-3 имеет близкие значения длительной прочности. Чуть более высокая длительная прочность в состоянии 1 объясняется более крупным размером $\gamma$ зерен и низкой объемной долей первичной $\gamma^{\prime}$-фазы. Для состояний 2 и 3 была получена близкая длительная прочность при напряжениях 700 - 900 МПа. Некоторое различие в размерах $\gamma$ зерен и выделений $\gamma^{\prime}$-фазы повысило значения длительной прочности состояния 3 при напряжениях ниже 700 МПа. С учетом свойств, полученных при растяжении, оптимальной деформационно-термической обработкой спла-

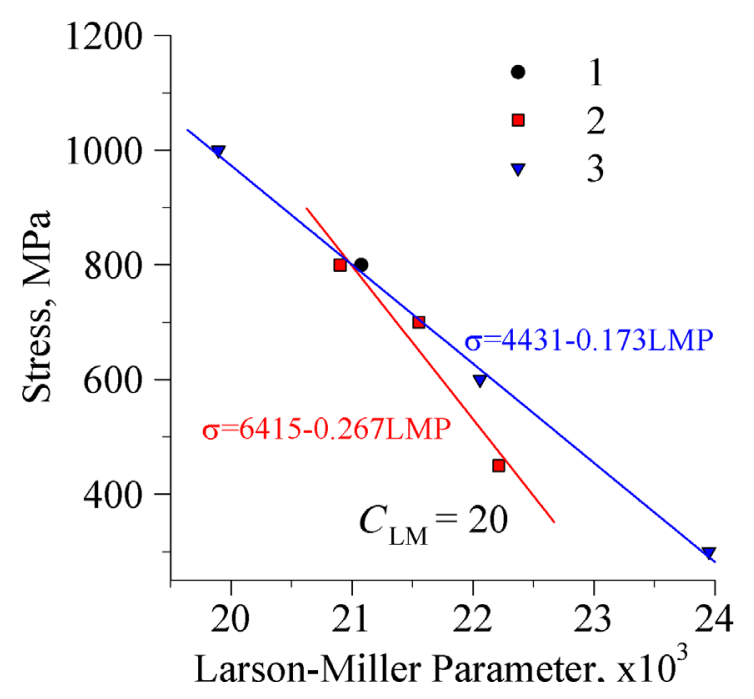

Pис. 4. (Color online) Параметр Ларсона-Миллера, построенный для состояний 1-3 сплава, испытанных на длительную прочность в диапазоне 650 - 850 $\mathrm{C} / 300-1000 \mathrm{MПа.}$

Fig. 4. (Color online) Larson-Miller parameter plotted for the superalloy in conditions $1-3$. The samples were subjected to long-term tests in the range of $650-850^{\circ} \mathrm{C} / 300-1000 \mathrm{MPa}$.

ва следует считать обработку в состоянии 3. В случае использования сплава в качестве штампового материала оптимальной обработкой следует считать обработку в состоянии 1 .

\section{4. Заключение}

В работе изучали новый экспериментальный никелевый сплав состава $\mathrm{Ni}-14(\mathrm{Al}, \mathrm{Ta})-30.7(\mathrm{Co}, \mathrm{Cr}, \mathrm{W}, \mathrm{Hf})$ (вес.\%), содержащий около 70 об.\% упрочняющей $\gamma^{\prime}$-фазы и имеющий высокую температуру полного растворения $\gamma^{\prime}$-фазы $\left(T_{\mathrm{s}} \approx 1260^{\circ} \mathrm{C}\right)$ благодаря высокому легированию танталом. Сплав показал повышенные или сравнимые значения предела текучести при сжатии в сравнении с традиционными никелевыми сплавами и может быть использован для изготовления штампового инструмента и роликов раскатного стана. Несмотря на присутствие небольшой объемной доли ТПУ фаз, новый сплав показал вполне приемлемые свойства при растяжении и длительной прочности в состоянии после горячей штамповки, обработки на твердый раствор и старения.

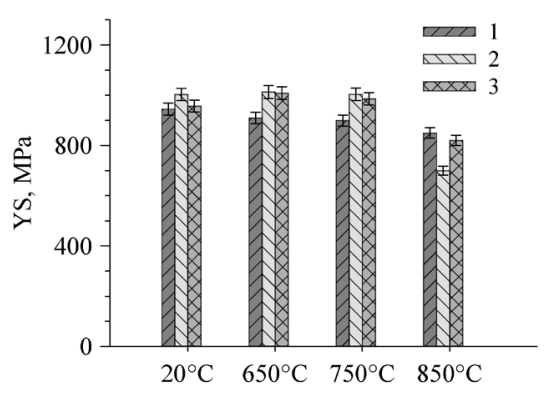

a

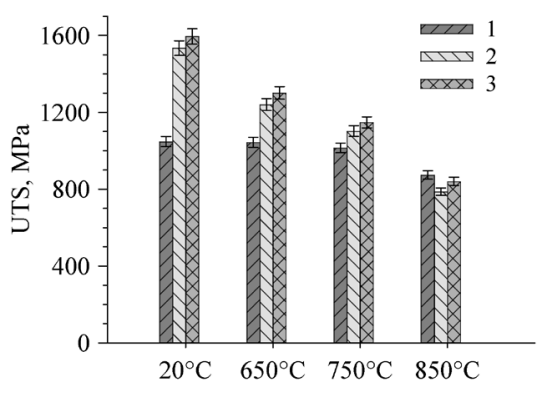

$\mathrm{b}$

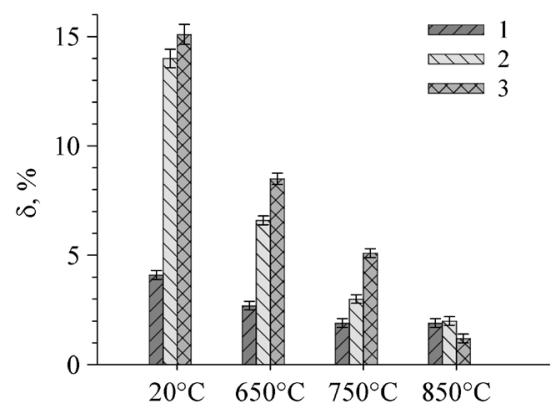

c

Рис. 3. Механические свойства сплава при растяжении в состояниях 1-3: предел текучести (YS) (a), временное сопротивление разрыву (UTS) (b), относительное удлинение $(\delta)(\mathrm{c})$.

Fig. 3. The tensile properties of the superalloy in conditions 1-3: yield strength (YS) (a), ultimate tensile strength (UTS) (b), elongation ( $\delta$ ) (c). 
Благодарности/Acknowledgments. Работа выполнена при поддержке Российского научного фонда (грант РНФ № 18-19-00594). Экспериментальные исследования были выполнены на базе Центра коллективного пользования научным оборудованием ИПСМ РАН. / The present work was supported by the Russian Science Foundation (Grant No. № 18-19-00594). The work was performed using the facilities of the shared services center "Structural and PhysicalMechanical Studies of Materials" at the Institute for Metals Superplasticity Problems of Russian Academy of Sciences.

\section{Литература/References}

1. R. C. Reed. The superalloys: Fundamentals and Applications. Cambridge University Press (2006) 372 p. Crossref

2. B. Geddes, H. Leon, X. Huang. Superalloys: Alloying and Performance. ASM International Materials Park (2010) 176 p. $\underline{\text { Crossref }}$

3. K. Sahithya, I. Balasundar, P. Pant, T. Raghu. J. Alloys Compd. 821, 153455 (2020). Crossref

4. O.A. Kaibyshev, F.Z. Utyashev. Superplasticity: Microstructural Refinement and Superplastic Roll Forming. Futurepast (2005) 386 p.

5. K. B. Povarova, O.A. Bazyleva, A. A. Drozdov, N.A. Alad'ev, M. A. Samsonova. Russian Metallurgy (Metally). 11, 975 (2012). Crossref

6. E. N. Kablov, O. G. Ospennikova, O.A. Bazyleva. Vestnik MGTU im N.E. Baumana. Ser. Mashinostroenie SP2, 13 (2011). (in Russian) [Е.Н. Каблов, О.Г. Оспенникова, О.А. Базылева. Вестник МГТУ им. Н.Э. Баумана. Сер. Машиностроение SP2, 13 (2011).]
7. R. I. Zainullin, A. A. Ganeev, R. V. Shakhov, A. V. Logunov, Sh. Kh. Mukhtarov, V. M. Imayev. Letters on Materials. 9 (4), 490 (2019). Crossref

8. F. R. Larson, J. Miller. Trans. ASME. 74, 765 (1952).

9. L.S. Mataveli, J. Cormier, P. Villechaise, D. Bertheau, G. Benoit, G. Cailletaud, L. Marcin. Mater. High Temp. 33, 361 (2016). Crossref

10. J. Radavich, D. Furrer. In: Superalloys 2004 (Ed. by K. A. Green,T.M. Pollock, H. Harada, T.E. Howson, R. C. Reed, J. J. Schirra, S. Walston). TMS, Warrendale PA, USA (2004) pp. 381-390. Crossref

11. O.G. Ospennikova. Razrabotka nauchnykh osnov sozdaniya novogo pokoleniya liteinykh jaroprochnykh nanostrukturirovannykh nikelevykh splavov ponijennoi plotnosti s trebuemym kompleksom mekhanicheskikh svoistv: Dissertacija na soiskanie stepeni doktora tehnicheskih nauk. Moscow (2018) 308 p. (in Russian) [О.Г. Оспенникова. Разработка научных основ создания нового поколения литейных жаропрочных наноструктурированных никелевых сплавов пониженной плотности с требуемым комплексом механических свойств: дисс. докт. техн. наук. Москва (2018) 308 c.]

12. L. Zheng, G. Schmitz, Y. Meng. Critical Reviews in Solid State and Materials Sciences. 37, 181 (2012). Crossref

13. G. A. Rao, M. Kumar, M. Srinivas, D.S. Sarma. Materials Science and Engineering A. 355, 114 (2003). Crossref

14. A. R. Braun, J.F. Radavich, C.P. Stinner. Superalloys 718 (Ed. by E. A. Loria). TMS, Warrendale PA, USA (1989) pp. 623-629. $\underline{\text { Crossref }}$ 